\title{
Providing Advice to Website Designers Towards Effective Websites Re-organization
}

\author{
Peter Tselios, Agapios Platis, George Vouros \\ University Of the Aegean, Department of Information and Communication Systems \\ Karlovassi, 83200, Samos, Greece \\ \{tpe, platis, georgev\}@aegean.gr
}

\begin{abstract}
This paper presents a method to help website designers to re-organize sites towards making pages that are "hidden" from site visitors but contain information that is of high importance for them, more accessible to future visitors. Towards this aim we present a method for computing the probability of visitors to be in a page and page's attractability. The computation of these indicators is based on transitions made to pages and on the information interest that the site designer assigns to pages. Based on this method, the paper presents DesignersAdvisor, which computes pages' attractability. DesignersAdvisor aims to assist the re-organization of any existing website without posing any additional effort to site visitors and without posing particular requirements concerning the form or the content of web pages.
\end{abstract}

\section{Introduction}

Web site designers structure their websites in a way that is comprehensive and more or less intuitive (according to designers' judgment) to the average visitor. However, it is very difficult to organize a site with a large number of pages in such a way that (a) every visitor could easily access information and (b) designers to achieve their communicative goals in the general case.

Our aim is to provide designers with tools that help them monitor visitors' behavior, help them to identify problematic cases in their sites and monitor the progress achieved towards making a "better" site organization. Problematic cases comprise information that website designers intent to present but, somehow, it is not easily accessible by website visitors.

Towards this aim we present a method that takes into account the activity of large number of people who have visited a website, calculates the probability of visitors to be in a page and pages' attractability. Attractability of a page is a new measure proposed here that depends on the probability of visitors to be in that page and the information interest that the site designer assigns to this page. The information interest captures in a simplistic way the communicative intention of website designers.

Based on the proposed method, we have developed DesignersAdvisor. This is a prototype tool that utilizes sites' log files in order to provide evidence for the behavior 
of websites visitors and to compute the attractability of pages based on designers' intentions. DesignersAdvisor assists the re-organization of any existing website without requiring any feedback from visitors and without posing particular requirements concerning the form or the content of web pages.

The paper is structured as follows: Section 2 sketches previous work towards reorganizing websites' structure for making information contained therein more accessible. It identifies the key issues of our research. Section 3 presents the proposed framework for modeling visitors, and describes the theoretical basis for the computation of the probabilities for visitors to be in a website page and pages' attractabilities. Section 4 presents DesignersAdvisor that implements the framework described in section 3, and provides the results of applying the proposed method to an existing website. Finally, section 5 concludes the paper and presents plans for future work.

\section{Related Work - Motivation}

Site re-organization aims at either customizing a website so as to satisfy the needs and characteristics of an individual user, or at optimizing (transforming, re-organizing) the structure of a site so as to make information more accessible and more effective to a large set of users. Furthermore, site re-organization may be content or access based [1]. In the first case re-organization decisions are based on pages' content, while in the second case they are based on requests of past visitors. The aim of this paper is to help designers of websites to structure their sites so as to make pages more accessible to future visitors, taking into account the activity of past visitors. This is an accessbased optimization method.

Our desiderata for a method for sites' re-organization are given in figure 1.

- It must not require additional effort from the visitors to provide feedback concerning their interest on pages' or sites prganization.

It must neither add/remove pages in the site automatically, nor alter the structure of the site by adding new pages Human webmasters must keep the control of the website structure.

- It must suggest web pages that have a high probability to be visited but are not found easily by sites' visitors.

It must take into account website designers' intents.

It must facilitate their easy integration into existing websites with the minimal effort from the webmasters.

Fig. 1. Desiderata for sites' re-organization method.

Systems that aim to transform the structure of websites in an access-based manner may be categorized according to their effect on browsing strategies within a site. According to $[13,14]$ there are three major browsing strategies:

a. Search browsing. In this case a user, to achieve specific goals, performs the same short navigation sequences relatively infrequently, but does perform long navigational sequences often" [13]. Systems that re-organize websites towards making them more effective to searchers include WebWatcher [7], and PageGather [2] [3]. WebWatcher [7] provides navigational support by predicting links that users may follow on a particular page as a function of their interests. This requires website visitors' feedback on their goal ("what they are looking for") and on whether they have found what they wanted. WebWatcher uses qualitative techniques since users 
state their interests in broad terms such as "accommodation facilities". On the other hand, PageGather proposes clusters of pages and synthesize index pages automatically. The system supposes that users visit a site with a particular information-seeking goal and therefore, pages that are interesting to them should be conceptually related. PageGather follows a statistical approach since it computes the co-occurrence frequencies between pages and creates a similarity matrix for pages.

b. General purpose browsing: In this case users consult sources that have a high likelihood of interest. As it has been computed in [13], probabilistically, users' behavior is more or less opportunistic. Efforts towards making websites more effective to general-purpose browsers include path traversal approaches such as Footprints [5] and the approach reported in [12]. Footprints computes visitors' access patterns. The basic idea is to visualize the paths that visitors have already followed and provide information concerning ways to proceed within the site. Footprints takes a statistical approach since it counts the number of users following the paths in a site. Similarly, the system reported in [12] computes path traversal patterns. The system computes frequent traversal patterns, i.e., large reference sequences. This is a statistical approach that takes into account the number of occurrences of reference sequences.

Based on evidence that a large class of website visitors, as already stated, do not repeatedly follow complex navigational patterns, our objective here is to emphasize on individual pages so as to make them more accessible to users. This is a complementary approach to finding path traversal patterns.

c. Serendipitous browsing: Users behavior in this case is purely random. Long invocation sequences are not repeated. Search engines are utilized to make websites more effective to serendipitous browsers. However, search engines do not assist websites' re-organization towards making them more effective to future visitors.

\section{Modeling Visitors and Attractability of Pages}

Based on our survey on access-based transformation approaches, it should be noted that most of them are statistical. For general-purpose and serendipitous human browsers that do not repeat navigational patterns and seek for interesting information, it seems that the evolution of their behavior in a website is Markovian. In this case, the transition from one page to another depends only on the information received from the former page. Based on this assumption, a probabilistic approach can provide the necessary information for making predictions on website visitors behavior based on pages' requests. Although this is true in the general case, such a model is not appropriate for sites where the navigational history plays a major role. For instance, this is not appropriate for educational hypermedia where the choice of the next page depends on the set of pages already visited.

Markov chain. Let $E$ be a set of points that represent the space of pages within a site and $X=\left\{X_{n}, n \in \mathbb{N}\right\}$ an E-valued stochastic process on a probability space, whose measure is $\operatorname{Pr}$ (with $\mathbb{N}$ with the set of positive integers). $X$ represents visitors' behavior evolution inside the space of web pages. If we assume that the transition from one page to another depends solely on the information contained in the former page, then 
$X$ is considered to be a Markov chain. That is, $\operatorname{Pr}\left\{X_{n+1}=j \mid X_{0}, X_{1}, X_{2}, \ldots, X_{n}\right\}=$ $\operatorname{Pr}\left\{X_{n+1}=j \mid X_{n}\right\}$ a.s., where $p(i, j)=\operatorname{Pr}\left\{X_{n+1}=j \mid X_{n}=i\right\}$ is called the transition probability from $\mathrm{i}$ to $\mathrm{j}$. Let $\alpha$ be the initial distribution on $E$. $X$ is completely defined by the initial distribution function $\alpha$ and the transition matrix $p$. Therefore, the probability of being in page $\mathrm{j}$ at time $\mathrm{n}$ is defined to be: $P_{j}(n)=\operatorname{Pr}\left(X_{n}=j\right)=\left(\alpha p^{n}\right)(j)$

The steady state probability distribution is given by $\lim _{n \rightarrow \infty} P(n)=\lim _{n \rightarrow \infty}\left(\alpha p^{n}\right)=\Pi$.

In this way, the probability of being in a page $i$ at an infinite time $n$, is given by

$$
\Pi_{\mathrm{i}}=\left[\lim _{n \rightarrow \infty}\left(\alpha p^{n}\right)\right](\mathrm{i})
$$

$\Pi_{i}$ gives the probability of being in page $i$ at a time where the evolution of website visitors behavior has reached a sort of stability. Furthermore, to infer those pages that are of real importance to visitors, based on website designers' intents, we introduce pages' information interest factor

Attractability: The attractability indicator is defined as a measure combining information on the probabilistic activity of website visitors and information concerning website designers' communication intents. Attractability has been derived from probabilistic performance indicators (e.g. in [10]).

Each web page $i$ in a site is associated with an "information interest factor" $\mathrm{g}(i)$ that represents the judgment of site designers on the importance of pages' information content. In this case, for instance, index pages are considered to have low information interest. We must notice that it is essential to take the designer's point of view and not to let the system evolve chaotically leaded only by the visitors' requests.

Given that the information interest factor assigned to each page is given by $\mathrm{g}(i)$ and that a site is observed in a time interval [n, $\mathrm{n}+\mathrm{N}]$ of length $\mathrm{N}$, where $n$ is large enough so that website visitors' behavior has reached a stability (given by steady state probabilities obtained by (1)), we can compute the attractability for each page on $[\mathrm{n}, \mathrm{n}+\mathrm{N}]$ in terms of requests made by websites' visitors for that page. So, we define the attractability of a page $i$ by:

$$
\text { atract }_{i}=g(i) \sum_{k=n}^{n+N-1} \operatorname{Pr}\left(X_{k}=i\right)=N g(i) \prod_{i}
$$

where $\sum_{k=n}^{n+N-1} \operatorname{Pr}\left(X_{k}=i\right)$ represents the total time spent in page $i$ in the time interval $[n, n+N]$ provided by means of (1). In this way, attractability of a page represents the cumulative reward that this page receives in time interval $[n, n+N]$.

Pages accessibility. While it is difficult in the general case to define a mechanism for promoting pages' links [1], we will use the term as a synonym to making pages more accessible. We define a page to be accessible wrt to its information interest when the (Percentage of requests)/(attractability) ratio of this page is equal to the corresponding ratio of all the pages in the site. Therefore, the proposed method reorganizes websites structure by combining, designers' intentions in terms of pages information interest and data coming from visitors' navigation in the site.

Towards finding pages that should be made more (less) accessible we propose finding (a) pages that have a percentage of visits lower (respectively, higher) than the mean value of the percentage of requests and (b) pages that have attractability greater 
(respectively, lower) than the mean value of pages attractabilities. Intersecting these two sets we obtain those pages that have low (respectively, high) percentage of visits and high (respectively low) attractability. This set of pages needs promotion (respectively, needs to be made less accessible).

\section{DESIGNERSADVISOR}

DesignersAdvisor is a prototype tool that utilizes sites' log files and computes the attractability of pages based on designers' intentions. DesignersAdvisor assists the reorganization of any existing website without requiring any feedback from visitors and without posing particular requirements concerning the form or the content of web pages.

The input to the DesignersAdvisor is the site's log file. The system preprocesses the log file and cleans it from useless information, such as requests to image files or error entries, and counts the number of transitions from page $i$ to page $j$ during a session. Based on indications provided by others $[6,13]$, we define a session to be a set of requests, where two subsequent requests are made within a time interval of 20 minutes. Using the number of transitions made from page to page, the system calculates the probabilities of visitors to be in each page of the site using (1), the attractability of each page using (2), and indicates the sets of pages that need to be made more/less accessible.

DesignersAdvisor has been applied to the ACAI '99 website log file. The log file contained almost 6000 valid records. These records represented requests to 41 pages made by 576 visitors. Figure 2 provides the information interest factor (which is an integer from 1 to100), the calculated probabilities, percentage of requests and attractabilities for some of the site pages. Let us for instance consider the page "Events". As it is shown in Figure 2, although this page has a small percentage of visits, the probability to be requested is relatively high. Furthermore, the attractability of this page is relatively high, which in conjunction with the number of requests indicates that this page needs to be made more accessible. It must be noticed that results provided by the system may be used (a) as a feedback to designers in order to reconsider their judgment concerning pages' information interest, and (b) to help them indicate the "problematic" cases in their site.

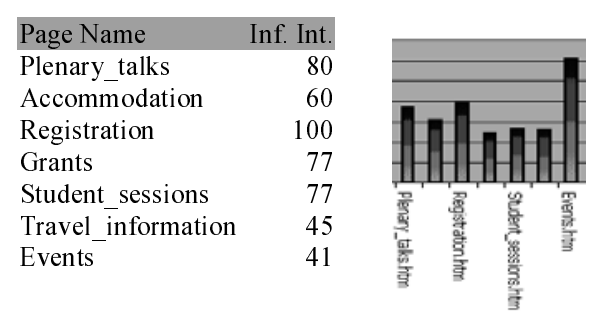

(a)

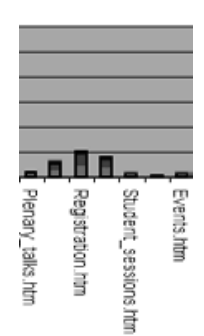

(c)

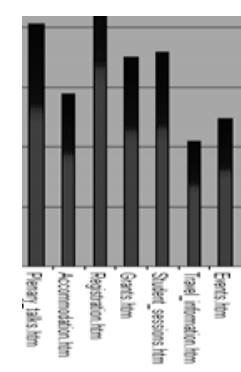

(d)

Fig. 2. (a) Pages' information interest, (b) probabilities, (c) percentage of requests and (d) attractabilities for some of the pages. 


\section{Conclusions - Future Work}

This paper presented a simple and effective access-based, probabilistic method, and a tool named DesignersAdvisor, to help website designers to re-organize their sites towards making information, which is of high importance and currently hidden, more accessible to future visitors.

Although this paper deals with single pages, the method could be extended to a subset of pages. In that case, the above method can be applied to clusters of pages, and give a clearer view of visitors' behavior in the site. Another issue that is subject to further investigation is the adequate representation of designers' intention. This paper does not provide any guidelines or any method concerning how information interest of pages should be assigned. Last, but not least, an empirical comparison with alternative methods needs to be carried in order to evaluate the provided results.

\section{References}

1. Perkowitz M., Etzioni O.: Adaptive Websites: an AI Challenge. In the proceedings of Fifteenth International Joint Conference on Artificial Intelligence (1997)

2. Perkowitz M., Etzioni O.: Adaptive Websites: Automatically synthesizing web pages. Proceedings of the Fifteenth National Conference on Artificial Intelligence, IAAA Press (1998)

3. Perkowitz M., Etzioni O.: Towards Adaptive Websites: Conceptual Framework and Case Study. To Appear in AI Journal.

5. Wexelblat A., Maes P.: Footprints: Visualizing Histories for web browsing. In proceedings of "5th RIAO Conference: Computer-Assisted Information Retrieval on the Internet", Montreal (1997)

6. Paliouras G., Papatheodorou C., Karkaletsis V., Tzitziras P., Spyropoulos C.D.: Learning communities of the ACAI ' 99 website visitors. In the proceedings of Advanced Course on Artificial Intelligence 1999 (1999)

7. Armstrong R., Freitag T., Joachims T., Mitchell T.: WebWatcher: A learning apprentice for the World Wide Web. In the proceedings of the 1995 AAAI Spring symposium on information gathering form heterogeneous, distributed environments, AAAI Press (1995), 612

10. Platis A., Limnios N., Le Du M.: Performability of electrical power systems modeled by non-homogeneous Markov Chains. IEEE Transactions on reliability, vol. 45 No. 4 (1996) 605-610

12. Chen M.S., Park J.S., Yu P.S.: Data mining for Path Traversal Patterns in a Web Environment. Proceedings of. 16th International Conference on Distributed Computing Systems, (1996), 385-392

13. L. Catledge and J. Pitkow, Characterizing Browsing Strategies. In: World Wide Web. In Proceedings of 3rd WWW conference, (1995)

14. Cove, J.F. and B.C. Walsh. Online text retrieval via browsing, Information Processing and Management, Vol. 24, No. 1, (1988) 31-37.

\section{Acknowledgements}

We would like to express our sincere thanks to the Software and Knowledge Engineering Laboratory of the Institute of Informatics and Telecommunications of the NRCS Demokritos for providing to us the log files of the ACAI'99 site. It should be indicated that the log files provided had no visitors' personal information. IPs were replaced by small integers. 